\title{
OBSERVAÇÕES SOBRE OS HÁBITOS E O COMPORTAMENTO ALIMENTAR DE PHR YNOPS GEOFFROANUS (SCHWEIGGER, 1812) EM CATIVEIRO (REPTILIA, TESTUDINES, CHELIDAE) ${ }^{1}$
}

Flavio de Barros Molina ${ }^{2}$

\begin{abstract}
Phrynops geofroanus is a carnivorous side-necked turtle. Its feeding behavior is divided in foraging, approach, capture, dilaceration and ingestion, but not necessary all these phases happen. Location of food seems to be visual and recognition olfactory. When food is an agile prey approach is done by pursuing and there is no olfactory recognition.
\end{abstract}

\section{INTRODUÇÃo}

Pouco se conhece sobre a histologia da maioria dos quelônios sul-americanos, sendo as espécies brasileiras ainda menos conhecidas que as do norte deste continente (Pritchard \& Trebbau, 1984). Isto é devido em parte, como apontam Carpenter \& Ferguson (1977), a associação da grande maioria das espécies a ambientes aquáticos ou semi-aquáticos, o que dificulta uma observação detalhada.

$\mathrm{Na}$ maioria dos casos o que se conhece a respeito do comportamento alimentar são aspectos de táticas especializadas, como as executadas pelo matamatá, Chelus fimbriatus.

As espécies do gênero Phrynops apresentam inúmeros problemas taxonômicos motivo pelo qual trata-se no presente estudo, como em Molina (1989), a espécie como Phrynops geoffroanus sensu Schweigger, 1812. A área de distribuição da espécie vai da Amazônia colombiana ao Rio Grande do Sul, Uruguai e norte da Argentina (Vanzolini et al., 1980), onde parece habitar preferivelmente lagoas e riachos a rios de maior volume (Medem, 1960 e Pritchard \& Trebbau, 1984), é predominantemente diurna e alimenta-se principalmente de peixes (Medem, 1960).

O presente estudo foi conduzido em cativeiro, onde as observações são mais facilmente realizadas, permitindo um detalhamento mais minucioso e tendo em vista ainda, o fato desta espécie mostrar-se muito arisca na natureza (Medem, 1960). Uma vez que o comportamento social e os concomitantes processos de comunicação entre répteis encontram-se bem desenvolvidos logo após o nascimento, devendo-se ter um considerável valor adaptativo (Burghardt, 1977), acredita-se como Lopes (1986) e Molina (1989) que o comportamento de adultos e sub-adultos provenientes da natureza pouco se alterará em condições adequadas de cativeiro. Além disso, o comportamento dos répteis mostrase em geral estereotipado (Carpenter \& Ferguson, 1977).

1. Parte da dissertação de Mestrado em Zoologia apresentada ao Instituto de Biociências da USP. Trabalho parcialmente financiado pelo CNPq.

2. Setor de Répteis da Fundação Parque Zoológico de São Paulo, Caixa Postal: 12954, CEP: 04092, São Paulo, SP. Brasil. 
De 1985 até a metade de 1989 estudou-se Phrynops geoffroanus na Fundação Parque Zoológico de São Paulo, São Paulo, SP, Brasil, buscando analisar-se vários tópicos relacionados a sua biologia e comportamento, com o objetivo de ampliar os poucos conhecimentos existentes, desenvolver um manejo adequado a sua criação em cativeiro e estudar a viabilidade da formação de um estoque de exemplares para possíveis repovoamentos (Molina, 1989). Este trabalho faz parte de uma série sobre a espécie.

\section{MATERIAL E MÉTODOS}

As observações realizadas junto ao acervo vivo do Setor de Répteis da Fundação Parque Zoológico de São Paulo abrangeram em média 30 machos e 40 fêmeas adultas de procedência ignorada e 135 filhotes nascidos em cativeiro (Molina, 1989).

Os adultos encontravam-se alojados em dois recintos a céu aberto, situados na área de visitação pública. Os recintos designados pelos números 78 e 81 continham respectivamente cerca de 25 machos e 35 fêmeas e 5 machos e 5 fêmeas.

$\mathrm{O}$ recinto 81 forneceu melhores condições de observação que o 78. Com cerca de $37 \mathrm{~m}^{2}$, possui no centro um tanque com profundidade máxima de $0,5 \mathrm{~m}$, área de $12 \mathrm{~m}^{2}$ e volume de $5.000 \mathrm{l}$, com um tronco parcialmente emerso em seu interior. A água é trocada a cada dois dias, o que permitiu observar os cágados no fundo do tanque, pelo lado externo do recinto, sem provocar interferências. Aqui conviveram com Phrynops geoffroanus exemplares de Chrysemys dorbignyi (tigre d'água) e Myocastor coypus (ratãodo-banhado).

O recinto 78 com cerca de $400 \mathrm{~m}^{2}$, possui na região central um tanque com profundidade máxima de $1 \mathrm{~m}$. Metade da área é arborizada, e constantemente sombreada, a outra metade encontra-se exposta ao sol. No tanque existem água-pés (Eichhornia crassipes) ou alfaces d'água (Pistia stratiotes) e em seu interior foi disposto tronco parcialmente emerso. A água do tanque é trocada a cada três meses. Neste recinto Phrynops geoffroanus conviveu todo tempo com exemplares de Geochelone carbonaria (jabuti), Chrysemys dorbignyi (tigre d'água), Hydromedusa tectifera (cágado), Caiman latirostris (jacaré-de-papo-amarelo) e Caiman crocodilus yacare (jacaré-do-Pantanal), e apenas parte do tempo com exemplares de Phrynops nasutus (cágado), Phrynops hilarii (cágado) e Caiman crocodilus crocodilus (jacaretinga).

A temperatura média mensal do ar nestes dois ambientes, oscilou de 16 a $28^{\circ} \mathrm{C}$ (extremos entre 6 e $35^{\circ} \mathrm{C}$ ).

A alimentação era oferecida três vezes, ou durante as épocas quentes do ano seis vezes por semana, sempre às 9:00 h. Os alimentos, sardinha picada e carne bovina moída (músculo) salpicados com farinha de osso, eram postos dentro d'água, bem distribuídos ao longo dos tanques. Tomate, cenoura, banana, mamão, verduras e tilápias inteiras (Oreochromis niloticus e Tilapia rendalli) eram oferecidos levando-se em conta as outras espécies que habitavam estes recintos.

Os filhotes encontravam-se alojados em tanques de fibro-cimento, com área seca e alagada, com dimensões respectivas de 0,48 a $0,71 \mathrm{~m}^{2}$ e 0,66 a 0,90 $\mathrm{m}^{2}$. A área alagada possuía $13 \mathrm{~cm}$ de profundidade, a área seca era formada por areia contendo alguma vegetação. Na água, trocada diariamente, havia ocasionalmente pés de alface d'água (Pistia stratiotes). Neste ambiente, os filhotes conviveram em grupos.

Por se encontrarem a céu aberto, os tanques eram cobertos à noite e durante fortes chuvas. Pelo mesmo motivo, quando a temperatura da água diminuía demais, en. 
Vol. 7(3), 1990

travam em funcionamento duas a três resistências do tipo utilizado em aquários (120 W/ $220 \mathrm{~V}$ ). A temperatura média mensal da água oscilou aproximadamente entre 20 e $30^{\circ} \mathrm{C}$ (extremos entre 15 e $35^{\circ} \mathrm{C}$ ), quando necessário o ambiente do tanque era aquecido por refletor com resistência elétrica $(250 \mathrm{~W} / 220 \mathrm{~V})$.

A alimentação era servida diariamente d̀s 9:00 h, dentro d'água. Como estudou-se o efeito desta no crescimento dos filhotes (Molina, 1989), estes receberam três tipos diferentes de alimentação. Dois grupos receberam sardinha moída salpicada com farinha de osso, periodicamente complementada por tilápia picada (Oreochromis niloticus e Tilapia rendalli) e invertebrados vivos, principalmente larvas de besouro da farinha (Tenebrio obscurus e Alphitobius piceus), ninfas e adultos de grilo (Gryllus sp). Dois outros receberam came bovina moida (músculo), sardinha moida, banana e mamão, tudo salpicado com farinha de osso, sendo que as frutas foram excluidas logo no início, por serem raramente aceitas. Três uutros grupos receberam ração para cães esfarelada.

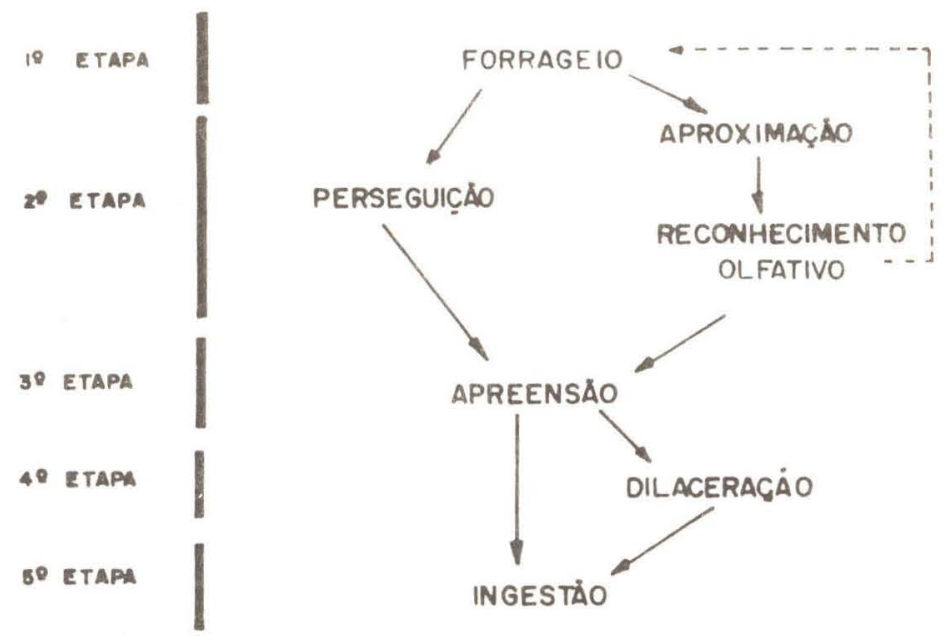

Fig. 1 - Diagrama da seqüència comportamental durante a alimentação. As setas cheias representam o fluxo comportamental e a tracejada, a possibilidade de interrupção e reinício.

\section{RESULTADOS}

O comportamento alimentar divide-se em cinco etapas sucessivas, nem todas obrigatórias: forrageio, aproximação, apreensão, dilaceração e ingestão do alimento (Figura 1).

Na primeira etapa, observada principalmente pela manhã, o indivíduo caminha. pelo fundo do tanque, ou nada próximo a este, com movimentos lentos, pescoço estica. do e cabeça mantida rente ao substrato (Figura 2A).

A segunda etapa tem início logo após a localização do alimento, que parece ser visual. Há uma aproximação, com movimentos lentos, se o alimento estiver imóvel (e.g. 
pedaços de carne e frutas) ou uma perseguição, se o alimento for uma presa viva dotada de agilidade (e.g., grilos e peixes). O pescoço continua esticado e a cabeça direcionada ao alimento. A aproximação pode ser feita em qualquer nível da água em que estejam os alimentos (figura 2B).

No final da segunda etapa ocorre o reconhecimento olfativo. $\mathrm{O}$ cágado aproxima as narinas do alimento (Figura 2C) e se este o interessar será apreendido. Caso o alimento não o interesse (e.g. frutas), voltará à primeira etapa. Quando a segunda etapa caracteriza-se como perseguição, não ocorre o reconhecimento olfativo.
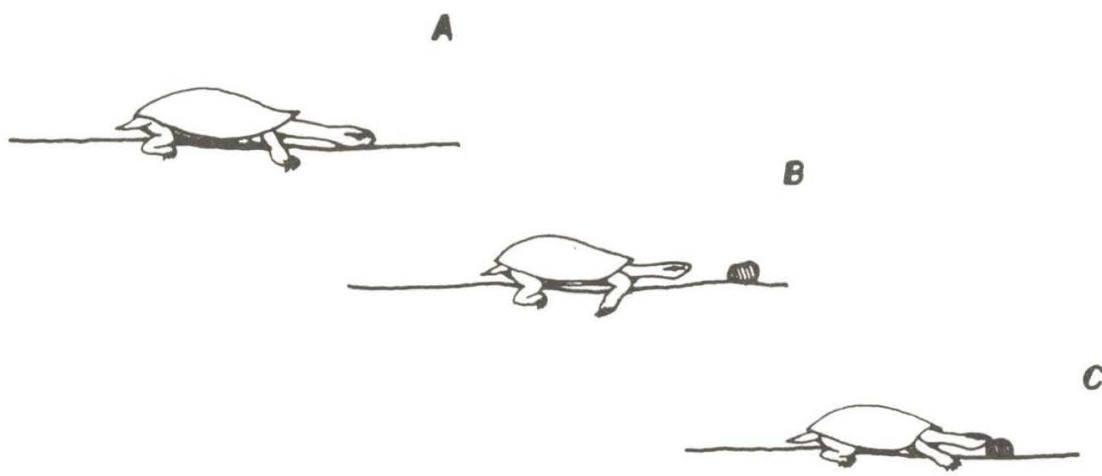

Fig. 2 - Etapas iniciais do comportamento alimentar. A) Forrageio, B) Aproximação e C) Reconhecimento olfativo.

A apreensão por sucção ocorre quando o alimento está dentro do raio de ação do cágado. $\mathrm{O}$ animal, normalmente com o pescoço não esticado, projeta a cabeça com um rápido movimento em direção ao alimento (Figura 3A), só abrindo a boca quando próximo a este e succionando-o (Figura 3B). A apreensão do alimento pode ocorrer em qualquer nível d’água.

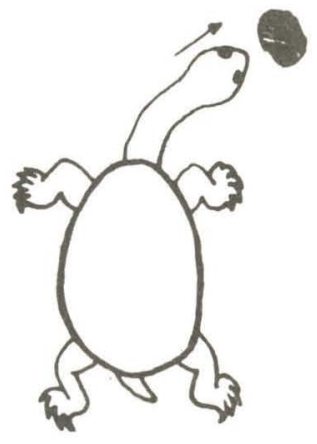

A

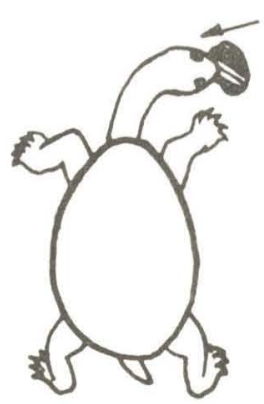

B

Fig. 3 - Etapas da apreensão do alimento. Em A, a cabeça é projetada em movimento rápido na direção do alimento; em B, a boca é aberta a fim de succioná-lo. 
Se o alimento for maior que a cabeça do cágado, terá que ser dilacerado antes da ingestão. A dilaceração é feita com auxilio das patas dianteiras, nunca utilizadas simultaneamente (Figura 4A). A pata utilizada é a que fica do lado para o qual a cabeça é recolhida, ao mesmo tempo que esta é retraída, a pata é afastada em arco, com as garras puxando parte do alimento (figura 4B). Os movimentos podem ser repetidos várias vezes, antes de ocorrer alteração da pata.

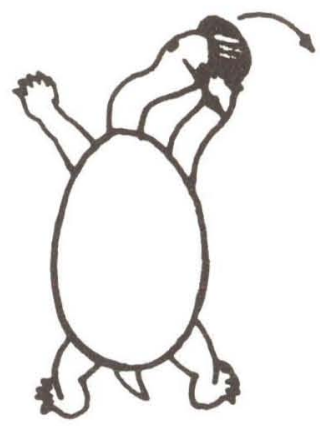

A

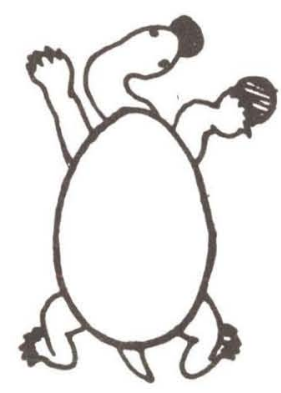

B

Fig. 4 - Etapas da dilaceração do alimento. Em A, a boca e uma das patas anteriores prendem o alimento; em B, ambas movimentam-se a fim de dilacerá-lo.

A ingestão ocorre por suç̧ão gradual, uma vez que ao fechar a boca o alimento tende a ser expelido. $\mathrm{O}$ cágado abre a boca várias vezes, succionando cada vez mais $\mathrm{O}$ pedaço abocanhado, até ingeri-lo por completo. Cada vez que fecha a boca ocorre expulsão da água succionada e restos de alimento (e.g. élitros de coleópteros).

Quando muitos indivíduos alimentam-se próximos, aquele que obtém um pedaço grande de alimento procura afastar-se, para não perdê-lo parcial ou totalmente. As etapas subseqüentes são finalizadas em local mais sossegado ou durante o afastamento, quase sempre perseguido pelos outros.

Disputas por alimento foram observadas entre exemplares de diferentes tamanhos e de outras espécies (Phrynops hilarii e Chrysemys dorbignyi). Nem sempre o maior indivíduo levou vantagem e as disputas só terminaram com a ingestão do alimento. Observou-se em algumas ocasiões, indivíduos morderem o pescoço de outros, o que pode não ter sido intencional, mas o resultado de um bote não certeiro.

Os adultos de Phrynops geoffroanus alimentaram-se bem de carne bovina moída e de sardinha em pedaços, praticamente não aceitando frutas, verduras ou outros vegetais. Os filhotes alimentaram-se de carne bovina moída e de peixe em pedaços, além de rações para cães, larvas de besouro da farinha e grilos. Ocasionalmente foi-lhes servido e também aceito, adultos de besouro da farinha, minhocas e pequenos guarús vivos. Grilos foram agilmente perseguidos e geralmente capturados, pequenos peixes só foram capturados quando o volume d'água no tanque encontrava-se bastante reduzido. Como os adultos, os filhotes praticamente não aceitaram vegetais. 
Revta bras. Zool.

\section{DISCUSSÃO}

Observações realizadas com outras espécies dulciaquicolas de quelônios neotropicais durante o desenvolvimento deste trabalho, mostram que o comportamento alimentar é bastante estereotipado para o grupo. Variações ontogenéticas não foram observadas, ao contrário do reportado por Froese \& Burghardt (1974) para Chelydra serpentina.

Das 13 espécies (incluídas em 9 gêneros e 4 famílias) observadas, 12 executam padrão que pouco difere do descrito para Phrynops geoffroanus, sendo Chellus fimbriatus a única excessão. Destas espécies, Phrynops hilarii, Acanthochelys spixii, Acanthochelys radiolata, Platemys platycephala, Hydromedusa tectifera e Hydromedusa maximiliani (todas da familia Chelidae) sã̃o as que exibem comportamento mais similar ao de Phrynops geoffroanus. As espécies dos gêneros Acanthochelys, Platemys e Hydromedusa por serem menos ágeis, caçam com mais dificuldade animais de movimentos rápidos, como grilos.

Fretey (ap. Pritchard \& Trebbau, 1984) observou Phrynops nasutus se alimentando por sucção e Pritchard \& Trebbau (1984) comentam que o extremo desenvolvimento do hióide nesta espécie, sugere adaptações paralelas às exibidas por Chelus fimbriatus. Outra espécie a executar comportamento semelhante ao de Phrynops geoffroanus é Chelydra serpentina (Chelydridae), segundo descrição de Froese \& Burghardt (1974).

Em espécies de outras famílias como Podocnemis expansa, Podocnemis unifilis e Podocnemis sextuberculata (Pelomedusidae), Rhinoclemys punctularia e Chrysemys. dorbignyi (Emydidae) e Kinosternon scorpioides (Kinosternidae), observou-se que a apreensão realiza-se principalmente pelo abocanhamento do alimento, sem a rápida projeção da cabeça como ocorre na família Chelidae. A sucção ocorre em grau menor, auxiliando apenas na ingestão de pequenas partículas, como apontado por Monge-Nájera \& Moreva-Brenes (1987) para Kinosternon scorpioides cruentatum. Para a dilaceração, Rhinoclemys punctularia, Chrysemys dorbignyi e Kinosternon scorpioidis, utilizam-se das patas dianteiras alternada ou simultaneamente. Pela disposição anatômica, as espécies da sub-ordem Pleurodira utilizam as patas alternadamente.

Não foi constatada neustofagia em Phrynops geoffroamus. Esta estratégia alimentar, descrita pela primeira vez por Belkin \& Gans (1968), consiste na ingestão de partículas alimentares em suspensão na superfície da água. Belkin \& Gans (1968) observaram neustofagia em Podocnemis unifilis e Chrysemys picta, Rhodin et al. (1981), estudando várias espécies das famílias Pelomedusidae e Chelidae, obsevaram esta técnica em $P_{O}$ docnemis unifilis, Podocnemis vogli, Podocnemis erytrocephala e Podocnemis expansa.

Dos quelônios neotropicais, o que mais se especializou quanto ao comportamento alimentar foi o matamatá, Chelus fimbriatus, que ao detectar a presa, dirige a cabeça vagarosamente a ela, esticando o pescoço. Repentinamente escancara a boca, com simultânea expansão da garganta, tragando assim a presa. Em seguida a boca é quase fechada, a água expelida e a presa ingerida (Bellairs, 1970; Pritchard, 1979; Pritchard \& Trebbau, 1984 e presente estudo).

Apesar de especializado na caça de espera, Chelus fimbriatus pode ao menos em cativeiro, exibir comportamento ativo, denominado por Holmstrom (1978) de pastoreio ("herding behavior"). Basicamente os peixes vão sendo conduzidos para a parte ıasa do tanque, onde são ingeridos. $O$ autor acredita que isto também possa ocorrer na natureza. 
A visão e o olfato, bem desenvolvidos nos quelônios em geral (Campbell, 1967; Bellairs \& Attridge, 1978 e Spellerberg, 1982), parecem ser os sentidos envolvidos no processo de procura, localização e reconhecimento do alimento em Phrynops geoffroanus. Estes sentidos são importantes na localização de alimento em Podocnemis unifilis (Belkin \& Gans, 1968) e algumas espécies do gênero Kinosternon (Mahmoud, 1968 e Hulse, 1974). Pritchard \& Trebbau (1984) sugerem que Chelus fimbriatus também possa utilizar a visão e o olfato na localização de alimento.

Seymour (1982) cita problemas relativos à alimentação na água, com instabilidade do corpo e dificuldade em fechar a boca sem expelir o alimento. Particularmente este último problema mostrou-se bastante evidente em Phrynops geoffroanus. Segundo Seymour (1982), tais problemas podem ser resolvidos pela movimentação dos membros enquanto o animal investe contra o alimento, ou pela rápida expansão da cavidade faríngea a fim de succionar o alimento.

Em todas as ocasiões em que o comportamento alimentar foi observado, ocorreu disputa pelo alimento entre indivíduos de Phrynops geoffroanus, ou entre estes e Phrynops hilarii e Chrysemys dorbignyi. Estudando jovens de Chelydra serpentina em cativeiro, Froese \& Burghardt (1974) observaram a existência de competição durante a obtenção de alimento. $\mathrm{O}$ grupo estudado apresentou uma hierarquia linear, que permaneceu estável por período de pelo menos vários meses. A posição ocupada pelo indiví. duo correlacionou-se positivamente com seu peso. Hierarquia linear também foi observada em espécimens de Emys orbicularis mantidos em cativeiro, onde a hierarquia mostrou-se dependente do grau de agressividade (boussekey, 1988).

Segundo Froese \& Burghardt (1974), determinados comportamentos podem ter a função de intimidar adversários, tais como o alongamento do pescoço ("neck stretching") e a sução do alimento ("snapping"). Indivíduos de Phrynops geoffroanus, e das demais espécies observadas da familia Chelidae, também exibiram estes comportamentos, não sendo possivel entretanto, analisar sua função intimidativa.

Phrynops geoffroanus em cativeiro, mostra-se predominantemente carnívoro, não havendo variações ontogenéticas a este respeito, como observado por Clark \& Gibbons (1969) e Hart (1983) para Pseudemys scripta. Segundo Medem (1960), na natureza Phrynops geoffroanus também se mostra carnívoro. Este autor encontrou restos de peixes em estômagos de vários exemplars e em um caso, asas de ortópteros e coleópteros, além de matéria vegetal. Em cativeiro Medem (1960) nunca observou a espécie alimentando-se de vegetais, mas sim de peixes, girinos, insetos aquáticos e minhocas. Segundo o Principe de Wied (ap. Goeldi, 1905) a dieta natural destes cágados parece constituir-se de pequenos peixes, moluscos, vermes e possivelmente vegetais aquáticos.

Segundo Medem (1966), outra espécie primariamente carnivora é Phrynops dahli, que na natureza alimenta-se de moluscos, insetos aquáticos, girinos, rãs, peixes e carniça. Em cativeiro, este autor observou que a espécie pode aceitar vegetais, afirmação feita por Mittermeier et al. (1978) para Phrynops gibbus. Fretey (ap. Pritchard \& Trebbau, 1984) observou que indivíduos de Phrynops gibbus em cativeiro, nunca aceitam matéria vegetal, o que também foi observado por Pritchard \& Trebbau (1984) para Phrynops nasutus.

\section{AGRADECIMENTOS}

À Dra. Norma Gomes (MZUSP) pela orientação da tese, aos Drs. Sérgio de Almeida Rodrigues, Miguel Trafaut Rodrigues e Ana Maria de Souza Oliveira (DZUSP) e ao 
meu irmão Rui de Barros Molina pela leitura crítica da mesma, aos meus pais e irmãos pelo encorajamento, aos colegas do Setor de Répteis do Zoológico de São Paulo pela colaboração, a Frederick José Pallinger pelos desenhos e a Maria Estela Frige pela datilograria do manuscrito.

\section{REFERÊNCIAS}

BELKIN, D.A. \& C. GANS, 1968, An unusual chelonian feeding niche. Ecology, 49 (4): 768-769.

BELLARIS, A., 1970. The life of reptiles. New York, Universe. v. 1, 282 p.

BELLARIS, A. \& J. ATTRIDGE, 1978. Los reptiles; trad. por José Luis Sanz Garcia. Madrid, H. Blume. $261 \mathrm{p}$.

BOUSSEKEY, M., 1988. Recherche expérimentale d'établissement d'une hiérarchie au sein d'un groupe captif de cistudes d'europe Emys orbicularis (Reptillia, Chelonii). Bull. Soc. Herp. Fr., (46): $1-9$.

BURGHARDT, G.M., 1977. Of iguanas and dinosaurs: social behavior and communication in neonate reptiles. Am. Zool., 17: 177-190.

CAMPBELL, H.W., 1967. The turtle in modern research. "How well will the turtle adapt to the moon's conditions?" Int. Turtle Tortoise Soc., 1:10-12.

CARPENTER, C.C. \& G.W. FERGUSON, 1977. Variation and evolution of stereotyped behavior in reptiles. In: GANS, C. \& D.W. TINKLE, eds. Biology of reptilia: Ecology and behavior. London, Academic Press. v. 7A, p. 335-554.

CLARK, D.B. \& J.W. GIBBONS, 1969. Dietary shift in the turtle Pseudemys scripta (Schoepff) from youth to maturity. Copeia, (4): 704-706.

FROESE, A.D. \& G.M. BURGHARDT, 1974. Food competition in captive juvenile snapping turtles, Chelydra serpentina. Anim. Behavi., 22 (3): 735-740.

GOELDI, E.A., 1905. Chelonios do Brazil. Bolm. Mus. para. E. Goeldi, 4:699-756.

HART, D.R., 1983. Dietary and habitat shift with size of redeared turtles (Pseudemys scripta) in a * southern Louisiana population. Herpetologica, 39 (3): 285-290.

HOLMSTROM, W.F., 1978. Preliminary observations on prey herding in the matamata turtle, Chelus fimbriatus (Reptilia, Testudines, Chelidae). J. Herpet., 12 (4): 573-574.

HULSE, A.C., 1974. Food habits and feeding behavior in Kinosternon sonoriense (Chelonia: Kinosternidae). J. Herpet., 8 (3): 195-200.

LOPES, H.R.., 1986. Biologia reprodutiva e comportamento do teiú Tupinambis tequixim (Linnaeus, 1758) em cativeiro (Reptilia, Teiidae). São Carlos, Universidade Federal de São Carlos. 131 p. (Tese de mestrado apresentada ao Departamento de Ciências Biológicas da UFSC).

MAHMOUD, I.Y., 1968. Feeding behavior in Kinosternid turtles. Herpetologica, 24 (4) : 300-305.

MEDEM, F., 1960. Informe sobre reptiles colombianos. (V) Observaciones sobre la distribucion geografica y ecologia de la tortuga Phrynops geoffroana ssp. en Colombia. Noved. Colombianas, 1 (5): 291-300.

MEDEM, F., 1966. Contribuciones al conocimiento sobre la ecologia y distribución geográfica de Phrynops (Batrachemys) dahli. (Testudinata, Pleurodira, Chelidae). Caldasia,9 (45): 467 489.

MITTERMEIER, R.A.; A.G.J. RHODIN; F. MEDEM; P. SOINI; M.S. HOOGMOED \& N. CARRILLO DE ESPINOZA, 1978. Distribution of the South American chelid turtle Phrynops gibbus, with observations on habitat and reproduction. Herpetologica, 34 (1): 94-100.

MOLINA, F.B., 1989. Observações sobre a biologia e o comportamento de Phrynops geoffroanus (Schweigger, 1812) em cativeiro (Reptilia, Testudines, Chelidae). São Paulo, Universidade de São Paulo. 185 p. (Tese de Mestrado apresentada ao Departamento de Zoologia do Instituto de Biociências da USP).

MONGE-NAJERA, J. \& B. MOREVA-BRENES, 1987. Notes on the feeding behavior of a juvenile mud turtle Kinosternon scorpioides. Herp. Review, 18 (1): $7-8$.

PRITCHARD, P.C.H., 1979. Ency clopedia of turtles. New Jersey, T.F.H., Neptune. 895 p.

PRITCHARD, P.C.H. \& P. TREBBAU, 1984. The turtles of Venezuela. Oxford, Society for the Study of Amphibian's and Reptiles. 403 p.

RHODIN, A.G.J.; F. MEDEM \& R.A. MITTERMEIER, 1981. The occurrence of neustophagia among podocnemine turtles. Br. J. Herpet., 6:175-176.

SEYMOUR, R.S., 1982. Physiological adaptations to aquatic life. In: GANS, C. \& F.H. POUGH, eds. Biology of Reptilia: Physiological ecology, special adaptations, energetics and growth. London, A cademic Press. v. 13, p. 1-51.

SPELLERBERG, I.F., 1982. Biology of reptiles: an ecological approach. Glasgow, Blackie \& Son Ltd. $158 \mathrm{p}$.

VANZOLINI, PE.; A.M.M. RAMOS-COSTA \& L.J. VITT, 1980. Répteis das caatingas. Rio de Janeiro, Academia Brasileira de Ciências. 161 p. 